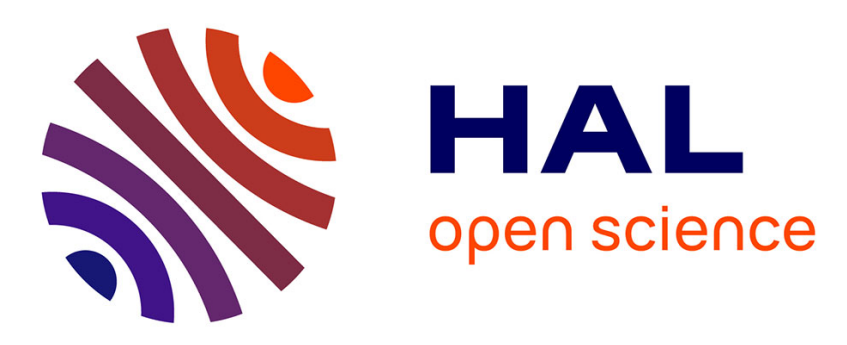

\title{
Automatic micromanipulation using multiscale visual servoing.
}

\author{
Brahim Tamadazte, Sounkalo Dembélé, Guillaume Fortier, Nadine Le \\ Fort-Piat
}

\section{- To cite this version:}

Brahim Tamadazte, Sounkalo Dembélé, Guillaume Fortier, Nadine Le Fort-Piat. Automatic micromanipulation using multiscale visual servoing.. 4th IEEE Internationale Conference on Automation Science and Engineering, CASE'08., Aug 2008, Key Bridge Marriott, Washington DC, United States. pp.977-982, 10.1109/COASE.2008.4626460 . hal-00324161

\section{HAL Id: hal-00324161 \\ https://hal.science/hal-00324161}

Submitted on 24 Sep 2008

HAL is a multi-disciplinary open access archive for the deposit and dissemination of scientific research documents, whether they are published or not. The documents may come from teaching and research institutions in France or abroad, or from public or private research centers.
L'archive ouverte pluridisciplinaire HAL, est destinée au dépôt et à la diffusion de documents scientifiques de niveau recherche, publiés ou non, émanant des établissements d'enseignement et de recherche français ou étrangers, des laboratoires publics ou privés. 


\title{
Automatic Micromanipulation using Multiscale Visual Servoing
}

\author{
Brahim Tamadazte, Sounkalo Dembélé, Guillaume Fortier and Nadine Le Fort-Piat \\ FEMTO-ST Institute, UMR CNRS 6174 - UFC / ENSMM / UTBM, \\ Automatic Control and Micro-Mechatronic Systems Department \\ 24 rue Alain Savary, 25000 Besançon, France \\ E-mails : \{btamadaz, sdembele, gfortier, npiat\}@ens $2 m$. fr \\ Web site : http://www.femto-st.fr
}

\begin{abstract}
The paper deals with the manipulation of silicon microcomponents in order to assembly automatically. The size of the components vary from $600 \mu \mathrm{m} \times 400 \mu \mathrm{m} \times 100 \mu \mathrm{m}$ to $300 \mu \mathrm{m} \times \mathbf{3 0 0} \mu \mathrm{m} \times \mathbf{1 0 0} \mu \mathrm{m}$ with a notch of $100 \mu \mathrm{m}$ thickness on every side. The microassembly process is split up into elementary tasks (aligning component, positioning component, centering component, opening gripper, ...) where every one is achieved by visual servoing. The control laws are of the type exponential or polynomial decrease of error according to the task. The performing of the latter has required the implementation of an effective tracking algorithm in combination with a depthfrom-focus technique in order to maintain the target in focus and to recover the distance between the gripper and the component. The process includes the adaptation of the video microscope magnification to the required resolution (coarse to fine servoings). A multiple scale modelling and calibration of the photon video microscope is performed. The picking and placing of above components were achieved : the errors of positioning are respectively $1.4 \mu \mathrm{m}$ in $x$ and $y$ and 0.5 degree in orientation.
\end{abstract}

\section{INTRODUCTION}

More and more companies are interested by automatic microassembly stations that are precise, repeatable, versatile and eventually flexible in order to manufacture small and medium series of hybrid MEMS or MOEMS. Some examples of that kind of micro components based products are micro-bearings, laser diodes or instrumented microgrippers. Examples of microassembly systems can be found in the following references [1], [3], [4], [5], [10], [11].

The criteria enabling above objectives are :

- the development of weak scale motion sources,

- the development of adequate kinematics combining wide scale motion and weak scale motion sources,

- the development of accurate feedback information sources of the work scene,

- the understanding of physical and chemical interactions at the microscale,

- the development of effective signal processing and control algorithms.

A lot of solutions have been proposed in the literature:

- Nelson and his teams in the USA and in the Switzerland, have developed many assembly station [1], [3], [10],

- Sun and Chin in China have presented a microassembly station, in which they used a multiple-view and multiple-scale approach by visual servoing for micromanipulation [2],

- Popa and al are very interested in the manipulation of the optical fibers, notably by image-based servoing [4], [6],

- Works concerned by microassembly station agility and flexibility are presented in the references [7], [8],

The main common feature to those stations is the use of multiple scale photon imaging systems as information sources. CCD or CMOS vision sensors are associated with lenses whose resolutions vary from low for wide scale motions to high for weak scale motions. Those systems often include at less two views : a top view for the positioning in the plane and a side view for the depth positioning. Then, automatic microassembly implies implementation of visual servoings paradigms taking into account the scale and view changing.

The paper deals with the problem of manipulating silicon MEMS devices. A sequential paradigm is proposed : the global task is split into elementary tasks where every one is achieved by visual servoing. The multiscale concept enabling coarse to fine positioning is also achieved sequentially in the visual servoing. Section II presents the properties of the video microscope and their effects on the manipulation. Section III describes the concept of micromanipulation process corresponding to the sequencing of elementary tasks. Section IV describes the control laws implemented in the visual servoings while section $\mathrm{V}$ describes the vision algorithms. Section VI presents the results obtained with a monoview multiple scale photon imaging system. The paper is ended by a conclusion and analysis of above concepts and results.

\section{Manipulating Under A Photon Video MICROSCOPE}

A photon video microscope includes a microscope associated with one or two cameras. The image quality is mainly determined by the microscope which is a combination of at least an objective (with $F_{0}$ the focal length) and a tubelength (with the length $T_{o p}$ ) (figure 2). The tube enables the obtaining of high optical magnification but makes the system cumbersome. However, the development optics allows today the obtaining of high magnification with compact system. 


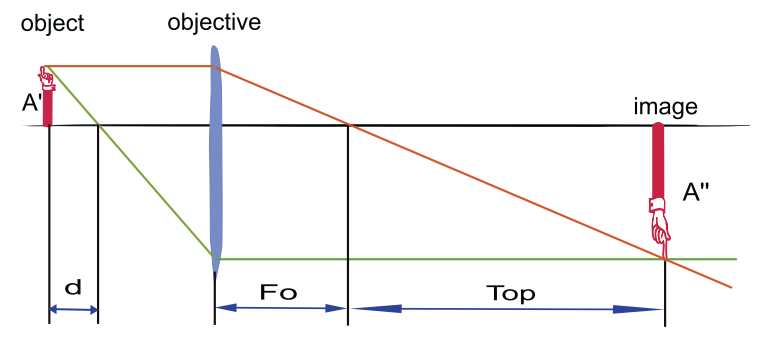

Fig. 1. Image formation principle with a photon microscope

The magnification $\Gamma$ is of the form :

$$
\Gamma=\left(T_{o p} / F_{o}\right)
$$

High magnification is obtained for very small focal length or great tube-length.

The depth of field DoF is :

$$
D o F=\left(n \cdot \lambda / N A^{2}\right)+(n \cdot e / \Gamma \cdot N A)
$$

with $\lambda$ the wavelength, $N A$ the numerical aperture, $n$ the refraction index of the medium (air in the case of that paper), $e$ the resolvable distance of image sensor.

The field of view $\mathrm{FoV}$ is :

$$
F o V=\left(T_{s} / \Gamma\right)
$$

with $\left(T_{s}\right)$ the sensor size.

The increasing of the magnification leads to the decrease of the field of view.

According to the value of the optical parameters, above formulas indicate that:

- the depth of field is very weak, about the value of the wavelength $(400 \mu m<\lambda<800 \mu m)$,

- the field of view is weak according to the value of the magnification.

Those properties complicate the automation of the manipulation, the latter requires the implementation of multiscale imaging (changes of magnification of zoom according to the task), of autofocus algorithm in order to get sharp images whenever the objects move in the work scene.

However those properties can be used to retrieve information from images using depth-from-focus methods like depth, shape or pose of objects.

\section{Modelling of the Manipulation Process}

The achievement of a manipulation task requires the achievement of some elementary tasks in a well determined order. The performing of any elementary task is possible if and only if the previous task is completed. As a consequence a manipulation process can be modelled by a sequence of tasks logically linked each other.

Let us consider the process of picking up and placing a microcomponent using a two-finger gripper and a monoview multiscale imaging system. The sequence of that process is:
- task 1 : autofocusing

- task 2 : detection of the component,

- task 3 : aligning of the component parallel to the axis,

- task 4 : positioning and centering of the component under the gripper,

- task 5 : opening of the gripper,

- task 6 : going down of the gripper,

- task 7 : closing of the gripper,

- task 8 : grasping of the component,

- task 8: going up of the gripper with the component,

- task 9 : positioning of the component,

- task 10 : going down of the gripper with the component,

- task 11 : opening of the gripper in order to release the component,

- task 12 : positioning of the gripper to the initial place.

It is represented as a chart in (figure 3 ). The tasks relative to motions are performed at different scales. The tasks 3 to 7 are performed at weak scale i.e. with high accuracy, the other tasks are performed at wide scale with low accuracy. The scale change is obtained by changing the magnification or zoom factor of the photonic imaging system.

Above motion tasks are achieved by $2 \mathrm{D}$ visual servoing approaches. The control laws are of the type exponential decrease of error for all the tasks except for task 7 where a polynomial decrease of error is implemented.

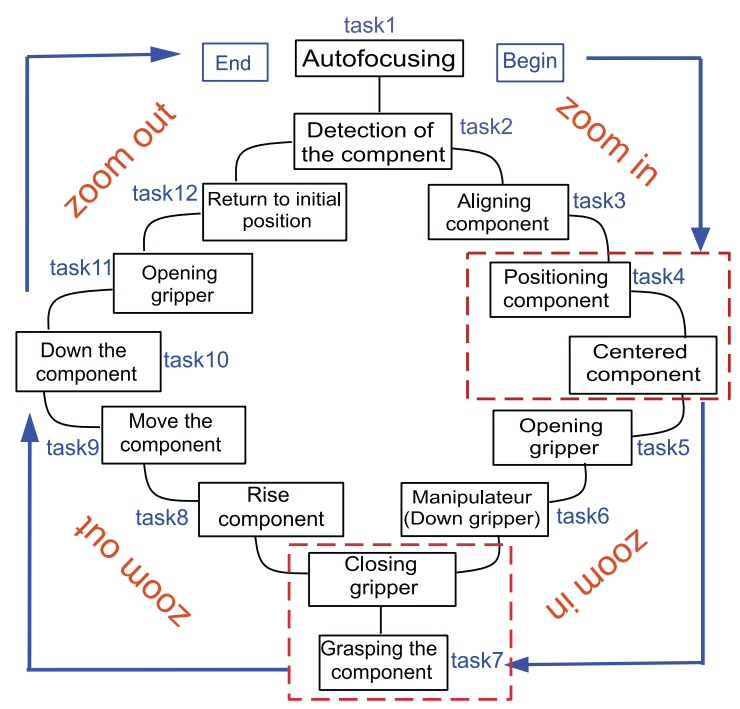

Fig. 2. Sequence chart of the picking and placing of a component

\section{VisuAl CONTROL LAWS}

1) Exponential Decrease of Error with Adaptive Gain: The tasks where several degrees of freedom have to be controlled are achieved by a visual servoing with a exponential decrease of error as exposed in the works of Chaumette and al [12], [13]. The multiple scale paradigm is introduced by explicitly modelling the scale factor and the focal length as functions of the magnification or zoom factor. 
Let $\zeta$ be the zoom factor. The multiscale calibration is performed that establishes the relation between the scale factor $k$ and the zoom factor $\zeta$. It is a polynomial relation of the form :

$$
k=\left[c_{1}, c_{2} \cdots c_{11}\right] \cdot\left[\zeta^{10}, \zeta^{2}, \cdots, 1\right]^{T}
$$

$c_{(1, \ldots, 11)}$ are the polynom coefficients.

As a consequence the focal length $f(f(\zeta))$ and the feature points $(s(\zeta))$ are functions of the zoom factor $\zeta$.

Let $s(\zeta)$ and $s^{*}(\zeta)$ respectively be the current and desired value of the set of selected visual feature points. The velocity $\dot{s}$ of those visual features are linked to the relative velocity of the camera/scene $v$ by the following equation :

$$
\dot{s}=L_{s}\left(s, Z_{f}, \zeta\right) v
$$

$L_{s}$ is the interaction matrix and $v=\left[v_{x}, v_{y}, v_{z}, w_{x}, w_{y}, w_{z}\right]$ is the kinematic screw. $Z_{f}$ represents depth information of the considered object expressed in the camera frame. That information is calculated by a depth from focus approach.

The interaction matrix for the coordinates $x$ and $y$ is respectively :

$$
\begin{aligned}
& L_{s}\left(x, Z_{f}, \zeta\right)= \\
& {\left[\begin{array}{cccccc}
\frac{-1}{Z_{f}} & 0 & \frac{x(\zeta)}{Z_{f}} & x(\zeta) y(\zeta) & -1-x(\zeta)^{2} & y(\zeta)
\end{array}\right]} \\
& L_{s}\left(y, Z_{f}, \zeta\right)= \\
& {\left[\begin{array}{llllll}
0 & \frac{-1}{Z_{f}} & \frac{y(\zeta)}{Z_{f}} & 1+y(\zeta)^{2} & -x(\zeta) y(\zeta) & -x(\zeta)
\end{array}\right]}
\end{aligned}
$$

Let $\mathrm{e}$ be the function task to regulate to zero :

$$
e=\left(s(\zeta)-s^{*}(\zeta)\right)
$$

The exponential decrease of the function task $\mathbf{e}$ :

$$
\dot{e}=-\lambda_{a} e
$$

leads to the following control law :

$$
v=-\lambda_{a} \widehat{L}_{s} e
$$

$\lambda_{a}$ is a positive gain. For a better convergence (good speed and without overshot) of $\mathbf{e}$, that gain is adapted to the value of e according to the following formula :

$$
\lambda_{a}=\lambda_{\max }+\lambda_{\min }-\alpha \log (e)
$$

where $\lambda_{\max }$ and $\lambda_{\min }$ are respectively the maximum and minimum values of $\lambda_{a}, \alpha$ is a constant.

That visual servoing approach can be represented by the functional chart given (figure 4).

2) Polynomial Decrease of Error: In that visual servoing only one degree of freedom is controlled. The control law consists in regulating toward zero the distance between the desired value $s_{f} *$ and the current value $s$ of a feature point.

Let the distance corresponding to the task function be $e_{1}$ :

$$
e_{1}=\operatorname{dist}\left(s_{f} *-s\right)
$$

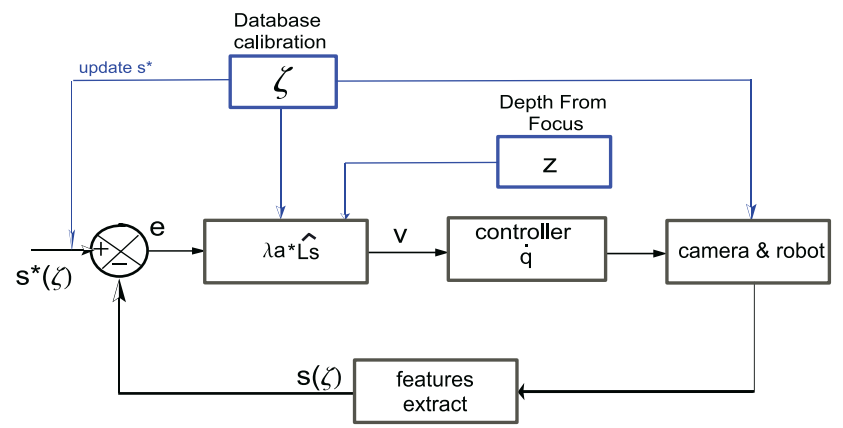

Fig. 3. The functional chart of visual servoing with exponential decrease of error

The polynomial decrease of that function leads to the following control :

$$
C_{d 1}(t)=\frac{e_{1}(t)}{N}
$$

Where $\mathrm{N}$ is constant. That control law $C_{d 1}$ is used for the control of one gripper finger (end-effector), for the other endeffector $-C_{d 1}$ is used. The motion of the two fingers are supposed symmetric. That visual servoing approach can be represented by the functional chart given in figure 5 .

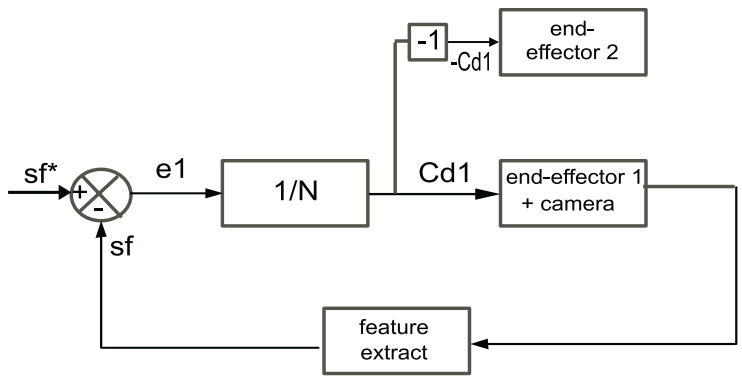

Fig. 4. The functional chart of visual servoing with polynomial decrease of error

\section{Vision Algorithms}

\section{A. Tracking of a Microcomponent and an End-effector}

Image processing and vision are very important in visual servoing. The following conditions are essential for the success of the control.

- Vision should be able to deliver visual informations at a rate near the sampling frequency of the robot control.

- Interaction matrix should be calculated explicitly.

- The command must be robust to little occultations (like the passage of the micro part under the gripper).

The images of the planar silicon microparts are not very sharp because of the properties of the microscope. Then a robust tracking algorithm able of working at real-time is required. There are several methods of that type which are usually classified into two groups. The first group consists in the track of local features like lines, segments, points, 
edges... [9]. The results of these techniques are highly depended of the quality of the images and remain very sensitive to feature detection [14]. The other group contains methods that perform a comparison between two frames in image sequences by minimizing an error based on the image brightness. Those methods take into account some parameters like motion, deformation or illumination between the two frames or the frame and a template.

The tracking method used in the paper is based on the " Efficient Second-Order Minimization" algorithm proposed by Malis and al. [14], [15]. The ESM has a very high convergence rate than other techniques which is an important property for real-time tracking. Because of the absence of texture in the images of the microparts, the tracking of the four points delimiting the bounding box of the micropart is associated with ESM.

\section{B. Autofocus Achievement}

Because of the weakness of the depth of field as exposed above, an autofocus method is implemented in order to guarantee the obtaining of focused images during the experiments. The depth of the scene is scanned step by step, images are acquired and the focus are estimated. The system is repositioned in the position where the focus estimation is highest. Three focus estimators have been tested : the variance $F_{\text {Var }}$, the intercorrelation $F_{A}$ and the Brenner gradient $F_{B}$. Yu sun and al have summered a lot of methods to calculate the focus [16].

The formulas are respectively :

$$
\begin{gathered}
F_{\text {Var }}=\frac{1}{H \cdot W \cdot \mu} \sum_{H} \sum_{W}(i(x, y)-\mu)^{2} \\
F_{A}=\sum_{H} \sum_{W} i(x, y) i(x+1, y)-\sum_{H} \sum_{W} i(x, y) i(x+2, y) \\
F_{B}=\sum_{H} \sum_{W}(i(x+2, y)-i(x, y))^{2}
\end{gathered}
$$

where $H$ and $W$ are respectively the image height and width and $\mu$ is the mean of image intensity.

The variance focus estimator is choosed because it makes a trade off between resolution and speed of calculation.

The representation of the focus estimation according to $z$ motion give two peaks (figure 6): the little peak corresponds to the gripper and the high peak corresponds to the platform with the component.

Therefore, it is possible to determine the depth $Z_{f}$ between the camera and the micropart:

$$
Z_{f}=Z_{o}-Z_{g}
$$

where $Z_{o}$ and $Z_{g}$ are respectively the position of the micro object and the gripper along the microscope axis.

That depth $Z_{f}$ is used to servoy the motion of the video microscope on the motion of the gripper (figure 7) .

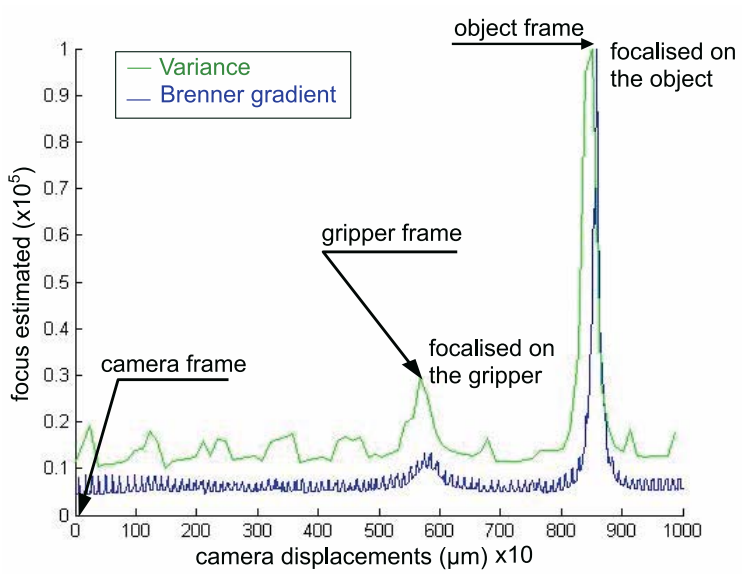

Fig. 5. The focus estimation result

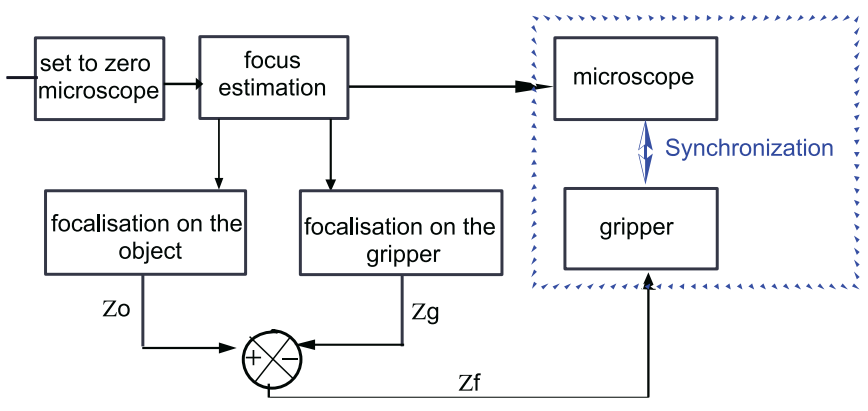

Fig. 6. Autofocus algorithm

\section{EXPERIMENTS}

\section{A. The Microassembly Station}

Above vision and control concepts have been validated on the microassembly cell developed in our lab with commercial positioning stages, video microscope and home made twofinger gripper and a compliant support (figure 8).
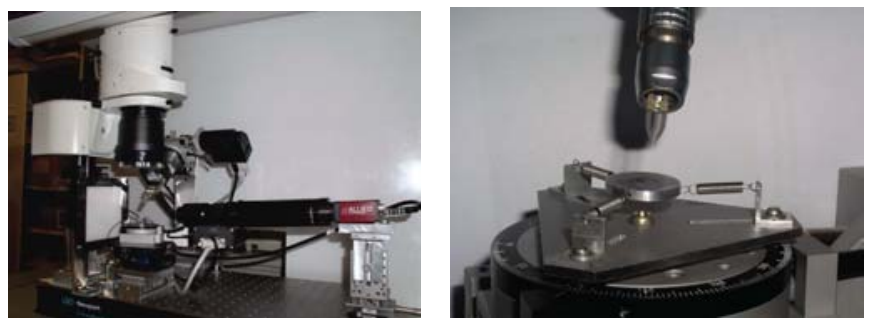

Fig. 7. Two views of the microassembly cell

In a kinematic point of view it is a 5 DOF robotic system. Three DOF are achieved by three high accuracy positioning stages : 2 linear stages forming a XY system (the resolution of every stage is $0.007 \mu \mathrm{m}$ ) and 1 rotating stage forming a $\theta$ stage (the resolution is $26 \mu \mathrm{rad}$ ). That system represents the $(\mathrm{XY} \theta)$ positioning platform unit. A linear stage and a rotating stage (the latter is positioned at 45 degrees according to the 
former) achieve the manipulating unit which supports the gripper. That gripper includes two fingers with 2 DOF for each one.

The imaging system is a video stereomicroscope of the type LEICA MZ 16 A vertically positioned to the work scene. Then, it delivers a top view of the work scene. The zoom (and then the magnification) and the focus are motorized and controlled by a PC. The field of view varies from $700 \mu \mathrm{m} \times 900 \mu \mathrm{m}$ with a resolution of $1.4 \mu \mathrm{m}$ at the maximum of the magnification to $20 \mathrm{~mm} \times 25 \mathrm{~mm}$ with a resolution of $21 \mu \mathrm{m}$ at the minimum of magnification. The depth of field varies from $2.9 \mathrm{~mm}$ to $0.035 \mathrm{~mm}$ according to the numerical aperture of the objective. The work distance is approximately $112 \mathrm{~mm}$.

The workcell is also equipped with a video microscope based on long tube for the side view but the latter is not considered in that paper.

\section{B. The Microcomponents to Handle}

The objects to manipulate are structures etched in silicon wafer. Following sizes are considered : $600 \mu \mathrm{m} \times 400 \mu \mathrm{m} \times 100 \mu \mathrm{m}$, $400 \mu \mathrm{m} \times 400 \mu \mathrm{m} \times 100 \mu \mathrm{m}$, $300 \mu \mathrm{m} \times 300 \mu \mathrm{m} \times 100 \mu \mathrm{m}$ (figure 9). They have a notche of $100 \mu \mathrm{m}$ enabling them to be assembled each other to form 3D structures.
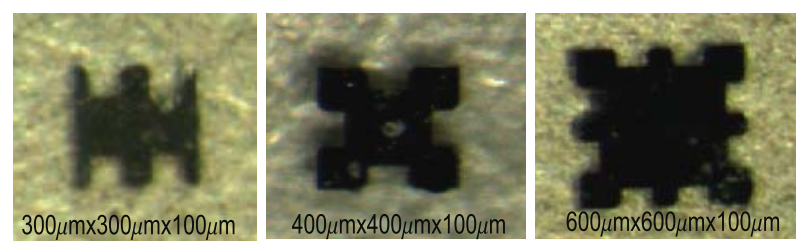

Fig. 8. The silicon microcomponents of experiments

\section{Picking and Placing the Microcomponents}

The sequence exposed at the section III is executed using the visual servoing approaches proposed at the section IV : a silicon microcomponent is picked up at an initial position and placed in a target position (figure 10). The images indicate the change of scale according to the required accuracy of the task.

Figure 11 shows the result of the task 3 i.e. the alignment of the component parallel to the $x$ axis. It can be see that the angle decreases exponentially to zero : the final error is about 0.5 degree. That very small value shows the relevance of the control law. It is possible to orientate effectively any planar component according to the axis.

Figure 12 shows the result of the task 4 i.e. the positioning of the component according to the gripper. It can be seen that the implementation of an adaptive gain prevents the overshoot of the reference position. The final positioning error is about $1.4 \mu \mathrm{m}$ in $x$ or $y$ directions.

Figure 13 shows the result of the task 7 corresponding to the closing of the gripper and the grasping of the component.

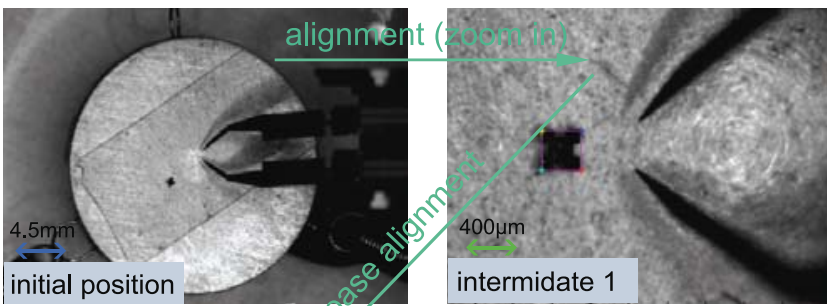

0
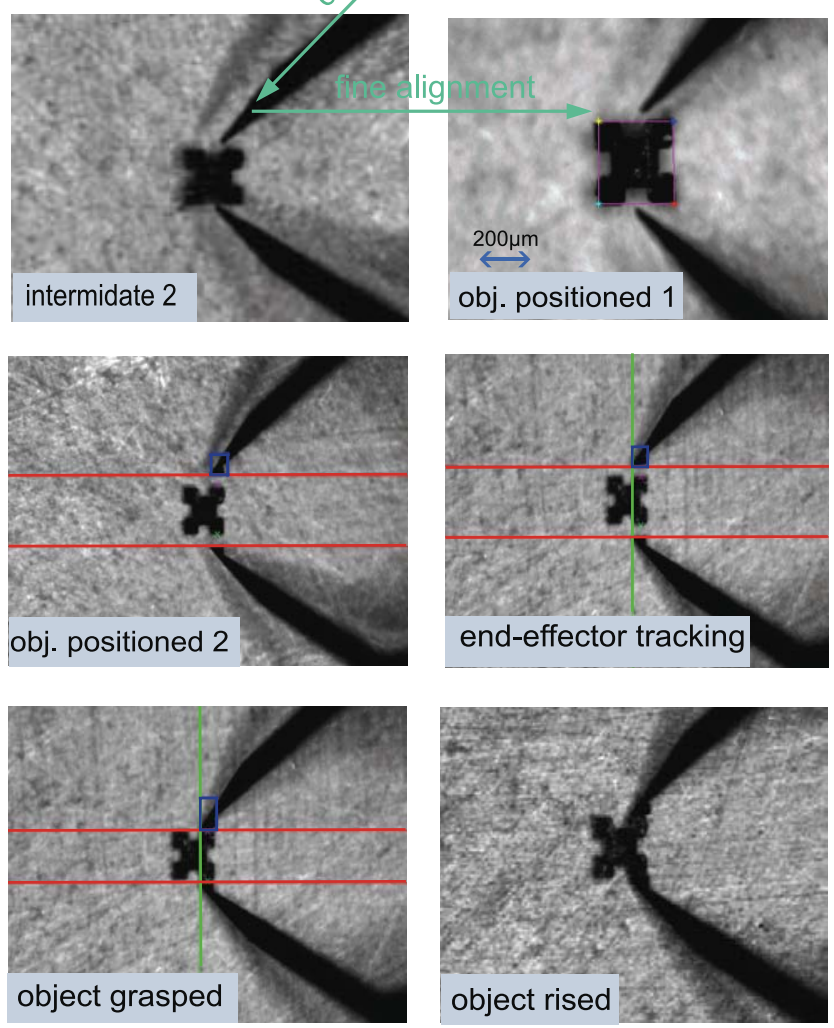

Fig. 9. Some shots during the studied sequence

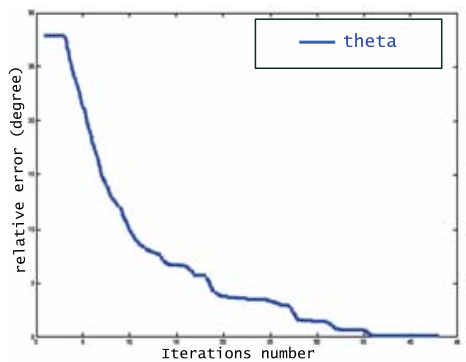

Fig. 10. The component alignment

The error ensures the grasping of the component in order to displace it. In that case the tracking is as exposed down. A small template image $W_{1}(n \times m)$ corresponding to the gripper tip is defined. Let $p_{1}=\left(u_{1}, v_{1}\right)$ be the $(2 \times 1)$ vector containing the coordinates of the left down pixel in that template and $p_{2}=\left(u_{2}, v_{2}\right)$ be the coordinates of the pixel belonging to the object selected to ensure a better grasping strategy of that object (figure 10). The purpose is 

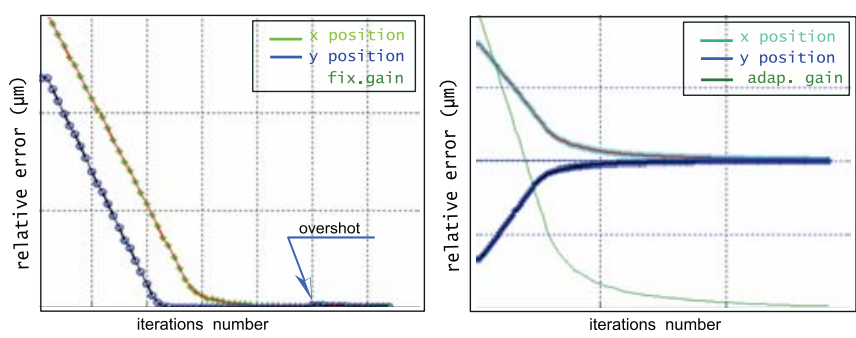

Fig. 11. The component positioning according to the gripper

to move $p_{2}$ into $p_{1}$. The control of the piezoelectric actuator of the gripper by application of a small control $C_{d 1}$ and $C_{d 2}$ prevents the large creeps and hysteresis.

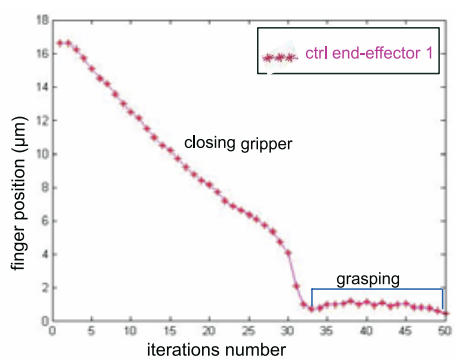

Fig. 12. The closing of the gripper and the component grasping

Experiments also show the robustness of the approaches to small occlusions of the object. The algorithm works when about $1 / 4$ of the object is out of the field of view or when the object passes under the gripper.

\section{CONCLUSION}

The problem of manipulating automatically micrometric components is studied.

The proposed idea is to model the process of manipulation by a sequence of tasks and to perform every task by a visual servoing. For every task, a control based on exponential decrease of error is proposed. A second type is proposed for simple task like soft closing of a gripper : a polynomial decrease of error. Those visual servoings include the change of the scale of manipulation as required by the tasks. For that purpose the model of the vision system integrating the magnification factor is developed.

Vision algorithms implemented to support above concepts include autofocus based on depth-from-focus approach and effective visual tracking in the images.

Experiments with a microassembly station have shown the relevance of above concepts. The components are aligned according to the $x$ axis with an accuracy of 0.5 degree, positioned according to the gripper with an accuracy of 1.4 $\mu \mathrm{m}$ in $x$ and $y$ directions without any overshoot. They are grasped softly.

Next work will deal with the accuracy of the whole process, particularly the final error between desired position and obtained position. That precision of the process depends on the control of interaction effects : humidity, thermal growth, the electrostatic forces... And finally automatic assembly of 3D structure will be conducted.

\section{AKNOWLEDGEMENTS}

This work is conducted with financial support from the project Hybrid Ultra Precision Manufacturing Process Based on Positional and Self assembly for Complex Micro-Products (HYDROMEL NMP2-CT-2006-026622) funded by the European Commission.

\section{REFERENCES}

[1] Stephen J. Ralis, B. Vikramaditya, and Bradley J. Nelson, " Micropositioning of a Weakly Calibrated Microassembly System Using Coarse-to-Fine Visual Servoing Strategies ", IEEE Transactions on Electronics Packaging Manufacturing, Vol. 23, No.2, April 2000.

[2] W.T. Sun, T.C. Chin, " Image-based Visual Servo for Micromanipulation : A Multiple-View and Multiple-scale Approach ", International Symposium on Micro-NanoMechatronics and Human Science, 2004.

[3] G. Yang, J. A. Gaines, and Bradley J. Nelson, " Optomechatronic Design of Microassembly Systems for Manufacturing Hybrid Microsystems ", IEEE Transactions on Industrial Electronics Vol.52, No. 4 August 2005.

[4] D. Popa, B. Hun Kang, J. Sin and J. Zou, " Reconfigurable Micro-Assembly System for Photonics Applications ", Proc. of ICRA in Washington, May 2002.

[5] Maysam Ghovanloo and Khalil Najafi," A Three-Dimensional Microassembly Structure for Micromachined Microelectrode arrays

", Proc. of Conf. on Microtechnologies in Medicine and Biology in Hawaii, May 2005.

[6] R. Muthy, A. Das, D. O. Popa, M. Mittal " M3: Multiscale Assembly and Packaging System for MOEMS ", Proc of SMTA Assembly Conf. and Expo, Chicago, September 2006.

[7] S. Koelemeijer Chollet and al, "A Flexible Microassembly Cell for Small and Medium Sized Batches ", Proc. of the 33rd ISR Oct. 2002.

[8] R. L. Hollis and A.A. Rizzi," Agile Assembly Architecture : A Platform Technology for Microassembly ", Proc. Am. Soc. for Precision Engineering 19th Annual Meeting, Orlando 2004.

[9] W Wang, X. Liu, and Y. Sun " Robust Contact Detection in Micromanipulation Using Computer Vision Microscopy ". Conf. Proc. IEEE Eng. Med. Biol. Soc. 2006, 22192222.

[10] Ge Yang, James A. Gaines, and Bradley J. Nelson, " A Supervisory Wafer-Level 3D Microassembly System for Hybrid MEMS Fabrication ", Journal of Intelligent and Robotic Systems 37: $43-68$ in 2003.

[11] Hui Xie, Weibin Rong and Lining Sun, " A Flexible Experimental System for Complex Microassembly under Microscale Force and Visual-Based Control ", International Journal of Optomechatronics, Nov. 2007.

[12] Eric Marchand, " Control Camera and Light Source Positions using Image Gradient Information ", Proc. of ICRA, Roma, Italy April 2007.

[13] François Chaumette and Seth Hutchinson "Visual Servo Control (PART I) ", IEEE Robotics and Automation Magazine, 13(4):82-90, Dec. 2006.

[14] Ezio Malis, " Improving Visio-Based Control Using Efficient Second-Order Minimization Techniques ", Proc. ICRA in New Orleans (USA) May 2004.

[15] Geraldo Silveira and Ezio Malis, "Real-time Tracking Under Arbitrary Illumination Changes ", In Computer Vision and Pattern Recognition, June 2007.

[16] Yu Sun, S. Duthaler and Bradley. J. Nelson, " Autofocusing Algorithm Selection in Computer Microscopy ", Proc. International Conference on Intelligent Robots and Systems, 2005. 